\title{
Plasma metabolite profiles in children with current asthma
}

\author{
R. S. Kelly ${ }^{1}$ (D) | J. E. Sordillo ${ }^{2}$ ｜ J. Lasky-Su ${ }^{1}$ | A. Dahlin ${ }^{1}$ | W. Perng ${ }^{3}$ | \\ S. L. Rifas-Shiman ${ }^{2}$ | S. T. Weiss ${ }^{1}$ | D. R. Gold ${ }^{1,4}$ | A. A. Litonjua ${ }^{5}$ | M.-F. Hivert ${ }^{2,6}$ | \\ \begin{tabular}{l|l} 
E. Oken & 2,7 \\
A. C. $\mathrm{Wu}^{2,8}$
\end{tabular}
}

${ }^{1}$ Channing Division of Network Medicine, Brigham and Women's Hospital, Boston, MA, USA

${ }^{2}$ Department of Population Medicine, Harvard Pilgrim Health Care Institute and Harvard Medical School, Boston, MA, USA

${ }^{3}$ Department of Nutritional Sciences, University of Michigan School of Public Health, Ann Arbor, MI, USA

${ }^{4}$ Department of Environmental Health, Harvard TH Chan School of Public Health, Boston, MA, USA

${ }^{5}$ Department of Pediatrics, University of Rochester, Rochester, NY, USA

${ }^{6}$ Diabetes Unit, Massachusetts General Hospital, Boston, MA, USA

${ }^{7}$ Department of Nutrition, Harvard TH Chan School of Public Health, Boston, MA, USA

${ }^{8}$ Division of General Pediatrics, Department of Pediatrics, Children's Hospital, Boston, MA, USA

\section{Correspondence}

Ann Chen Wu, Department of Population Medicine, Boston, MA, USA.

Email: ann.wu@childrens.harvard.edu

Funding information

National Institutes of Health, Grant/Award Number: K24 HD069408, R01 HD034568, R01Al102960, R01HD085993,

R01HL123915, U01HL091528

\section{Summary}

Background: Identifying metabolomic profiles of children with asthma has the potential to increase understanding of asthma pathophysiology.

Objective: To identify differences in plasma metabolites between children with and without current asthma at mid-childhood.

Methods: We used untargeted mass spectrometry to measure plasma metabolites in 237 children (46 current asthma cases and 191 controls) in Project Viva, a birth cohort from eastern Massachusetts, USA. Current asthma was assessed at mid-childhood (mean age 8.0 years). The ability of a broad spectrum metabolic profile to distinguish between cases and controls was assessed using partial least squares discriminant analysis. We used logistic regression models to identify individual metabolites that were differentially abundant by case-control status. We tested significant metabolites for replication in 411 children from the VDAART clinical trial.

Results: There was no evidence of a systematic difference in the metabolome of children reporting current asthma vs. healthy controls according to partial least squares discriminant analysis. However, several metabolites were associated with odds of current asthma at a nominally significant threshold $(P<.05)$, including a metabolite of nicotinamide (N1-Methyl-2-pyridone-5-carboxamide (Odds Ratio $(\mathrm{OR})=2.8(95 \% \mathrm{Cl} 1.1-8.0))$, a pyrimidine metabolite (5,6-dihydrothymine $(\mathrm{OR}=0.4$ (95\% Cl 0.2-0.9)), bile constituents (biliverdin ( $\mathrm{OR}=0.4$ (95\% $\mathrm{Cl} 0.1-0.9)$, taurocholate $(\mathrm{OR}=2.0(95 \% \mathrm{Cl} 1.2-3.4))$, two peptides likely derived from fibrinopeptide A (ORs from 1.6 to 1.7), and a gut microbiome metabolite ( $p$-cresol sulphate $\mathrm{OR}=0.5$ (95\% Cl 0.2-0.9)). The associations for N1-Methyl-2-pyridone-5-carboxamide and $\mathrm{p}$-cresol sulphate replicated in the independent VDAART population (one-sided $P$ values $=.03-.04$ ).

Conclusions and Clinical Relevance: Current asthma is nominally associated with altered levels of several metabolites, including metabolites in the nicotinamide pathway, and a bacterial metabolite derived from the gut microbiome. 


\section{KEYWORDS}

asthma, bile constituents, children, endogenous steroids, fibrinopeptides, glycerophospholipid

metabolism, metabolomics, nicotinamide synthesis, p-cresol sulphate, pyrimidine metabolism

\section{1 | INTRODUCTION}

Asthma is the most common chronic illness in U.S. children, and results in substantial morbidity as well as health care costs, with over $\$ 50$ billion dollars spent annually. ${ }^{1}$ Asthma exacerbations are the most common health-related cause of lost school days, ${ }^{1}$ rendering asthma a considerable public health burden. Asthma is a heritable disease with both environmental and genetic components. ${ }^{2,3} \mathrm{~A}$ number of molecular determinants have been identified for asthma, ${ }^{4}$ yet much remains unknown about how these molecular variants impact the disease. Metabolomics, the systematic analysis of all metabolites in a biological system, including carbohydrates, peptides, amino acids, organic acids, nucleotides and lipids, has emerged as a powerful tool to identify new biomarkers for several diseases, and may help identify novel pathways associated with complex disease. Metabolite fluctuations may be more accurate disease markers than transcriptional, translational or post-translational changes, as they represent an integrated pathophysiologic profile that captures genetic and environmental interactions. Changes in metabolite profiles associated with chronic disease status in epidemiological studies may highlight important metabolites or metabolic pathways to interrogate in both in vitro and in vivo studies of underlying pathophysiological mechanisms.

Investigators have successfully identified metabolite biomarkers for Type 2 Diabetes, Alzheimer's disease and cardiovascular disease, ${ }^{5-8}$ leading to the discovery of novel disease pathways for these conditions. Existing asthma metabolomic studies $^{9-22}$ have reported promising findings, identifying biologically plausible metabolites related to tricarboxylic acid (TCA) metabolism, hypermethylation, phospholipid regulation, hypoxic and oxidative stress, immune reaction and inflammation that have been associated with asthma and asthma severity. Nevertheless, the studies to date are limited by diagnostic heterogeneity, sample size, and number of metabolites, and most findings are yet to be replicated in independent populations. As such, the use of metabolomics in asthma studies remains in the early stages with much knowledge to be gained.

The goal of this study is to identify differences in metabolomic profiles for children with and without a current diagnosis of asthma, using an untargeted metabolomic profiling approach. We sought to determine whether children with current asthma have altered metabolic profiles as compared to controls without current asthma. Our goals were to (i) determine the ability of a broad spectrum metabolic profile to discriminate between current asthma cases vs. controls (ii) identify specific individual metabolites associated with current asthma and (iii) validate these findings using an independent population

\section{METHODS AND MATERIALS}

\subsection{Study population}

\subsection{1 | Discovery population}

Project Viva is an ongoing longitudinal pre-birth cohort study of children recruited from Atrius Harvard Vanguard Medical Associates, a multispecialty group practice in eastern Massachusetts. The purpose of Project Viva is to study the effect of environmental exposures on maternal and child health. Details of the study design and recruitment have been previously reported. ${ }^{23}$ The Institutional Review Board of Harvard Pilgrim Health Care approved the study protocols. All mothers participating in the study provided written informed consent and the children provided verbal assent. A total of 1,116 mother-child pairs (with children aged 6-10 years) attended an inperson mid-childhood study visit and 648 assenting children provided fasting blood samples. Metabolomic assays were originally performed for a study of childhood obesity and metabolomics in Project Viva. ${ }^{24}$ Given funding limitations, we selected 300 children for analysis, deliberately oversampling for maternal and child for obesity. Subjects were oversampled for obesity as the original analysis was focused on studying obesity. ${ }^{24}$

After further exclusion of 38 children with inadequate plasma volume for the metabolomic assays, 262 children (84 obese, 28 overweight and 150 normal weight participants) had metabolomic data. Of 262, we excluded from this analysis 21 children with past asthma diagnosis, and four with inadequate information on current asthma. Thus, the final analytic sample included 237.

We assessed current asthma during the mid-childhood visit (mean age 8.0 years). We defined current asthma in mid-childhood as maternal report of ever diagnosed with asthma by a healthcare professional (assessed on the mid-childhood questionnaire) plus either taking asthma medications in the past 12 months or wheezing symptoms the past 12 months. This analysis included 46 cases with current asthma, and 191 controls who had never reported a past asthma diagnosis, and who did not report wheezing or use of asthma medications in the past 12 months. We also collected information from in-person interviews and questionnaires on maternal age at enrollment, maternal education (a marker of socio-economic status), smoking during pregnancy and child age, gender and race/ethnicity. Information on asthma medication use and asthma severity was also collected from in-person interviews and questionnaires. Height and weight data collected at the mid-childhood visit were used to compute BMI z-scores based on CDC 2000 age and gender-specific reference data. ${ }^{25}$ Allergen sensitization was defined as any specific IgE 
level of $0.35 \mathrm{IU} / \mathrm{ml}$ or greater to common indoor allergens (Dermatophagoides farinae, cat, dog and cockroach), mold allergens (Alternaria or Aspergillus species), food allergens (egg white, milk and soy bean), or outdoor allergens (rye grass and ragweed).

Plasma metabolomic profiling for Project Viva was performed at Metabolon $^{\mathrm{TM}}$ for 262 children based on availability of archived biospecimens for a separate study of metabolomics and obesity status. ${ }^{24}$ Untargeted metabolomic profiling was performed using multiplatform mass spectrometry (MS); detailed descriptions of sample preparation and metabolite identification procedures have been previously published (see Appendix S1 for additional details). ${ }^{26}$ Samples were prepared using the automated MicroLab STAR ${ }^{\circledR}$ system from Hamilton Company for both Project Viva and VDAART. Several recovery standards were added prior to the first step in the extraction process for QC purposes. The resulting extract was divided into four fractions: one for analysis by UPLC/MS/MS (positive mode), one for UPLC/MS/MS (negative mode), one for GC/MS and one for backup. Samples were placed briefly on a TurboVap ${ }^{\circledR}$ (Zymark) to remove the organic solvent. Each sample was then frozen and dried under vacuum. Samples were then prepared for the appropriate instrument, as described below.

Briefly, the analysis was performed using three platforms: UPLC/ MS/MS (positive mode), UPLC/MS/MS (negative mode) and GC/MS, with pooled samples included throughout the analytical run for quality control purposes. Raw data were extracted, peak-identified and quality-control processed using Metabolon Inc. ${ }^{\circledR}$ hardware and software. Semi-quantitative concentrations (expressed in arbitrary units) of 345 known biochemicals were obtained. Metabolites were identified by comparison to library entries of purified standards or recurrent unknown entities. We included both endogenous and exogenous metabolites in our analyses. Missing values were imputed with half of the minimum value for that compound. Metabolite intensities were log transformed to normalize them and pareto-scaled to reduce the variation in fold-change differences between the features. See Appendix S1 for full details of the Metabolomic Profiling Procedures.

\subsection{2 | Replication population}

For replication of our findings in Project Viva, we used plasma samples of children born to mothers enrolled in the Vitamin D Antenatal Asthma Reduction Trial (VDAART) clinical trial; a two arm, doubleblind, placebo controlled, randomized, clinical trial of Vitamin D, to determine whether higher vitamin $D$ intake and levels in the pregnant mother will prevent asthma and allergy in childhood. ${ }^{27}$ Pregnant women (who had or whose partner had allergies/asthma) were randomized ( $n=881$ ) during the first trimester of pregnancy (1018 weeks) to one of two treatment arms of a clinical trial: 4000 IU Vitamin D+ prenatal vitamins or 400 IU Vitamin D+ prenatal vitamins. Of the 810 live births, 411 of had non-fasting plasma samples available at age three years for metabolomics analysis (108 asthma cases, 303 controls). Metabolomic profiling for VDAART was performed at Metabolon ${ }^{\mathrm{TM}}$, using a platform similar to the one utilized for Project Viva (see Appendix S1 for details). We used physician's diagnosis of asthma to define cases in VDAART at age three followup. ${ }^{28}$ Parental report of physician's diagnosis of asthma was taken directly from the offspring questionnaires.

\section{2 | Statistical analysis}

Analyses were conducted in R. $^{29}$ Differences in maternal and child demographic characteristics between cases and controls were assessed using $t$-tests for continuous variables and chi-square tests for categorical variables.

\subsection{1 | Partial least squares discriminant analysis}

Metabolite features were analysed as measured LC-MS peak areas, which are proportional to feature concentration and can be compared for any given metabolite (relative quantitation). Partial least squares discriminant analysis (PLS-DA) was conducted using MetaboAnalyst v.2.5 (http://www.metaboanalyst.ca/) ${ }^{30}$ to determine the combined ability of the 345 identified metabolites to discriminate asthma cases from controls. PLS-DA identifies latent factors, or components, that best describe the relationships between the metabolites, while best predicting asthma status. A sevenfold internal cross-validation procedure was implemented to guard against model over-fitting, and the overall significance of the model's discriminatory ability was evaluated using permutation testing; specifying 'prediction accuracy during training' and 2000 permutations.

\subsection{2 | Individual metabolite analysis}

We also conducted logistic regression on individual metabolites, to determine their relationship to asthma case status. Models were adjusted for maternal age, maternal education, smoking during pregnancy, child's gender, age, BMI z-score and race/ethnicity. Associations were expressed as odds ratios $(95 \% \mathrm{Cl})$ for current asthma per unit increase in metabolite.

\subsection{3 | Replication of significant findings}

For replication, metabolites significantly associated with asthma in Project Viva were tested in an independent cohort of children from the VDAART clinical trial. We performed logistic regression analysis to determine if these metabolites were associated with asthma diagnosis at age 3 years. Analyses were adjusted for child's age, gender, $\mathrm{BMI}$, race/ethnicity, maternal education level and maternal age. We report one-sided $P$ values for the replication analysis.

\section{3 | RESULTS}

\subsection{Discovery population}

Characteristics of the discovery population are presented in Table 1. Of the 237 children in Project Viva, 52.3\% were female and $19.4 \%$ had a mother-reported a diagnosis of current asthma. The mean 
TABLE 1 Demographics of discovery population, project viva $(\mathrm{N}=237)$

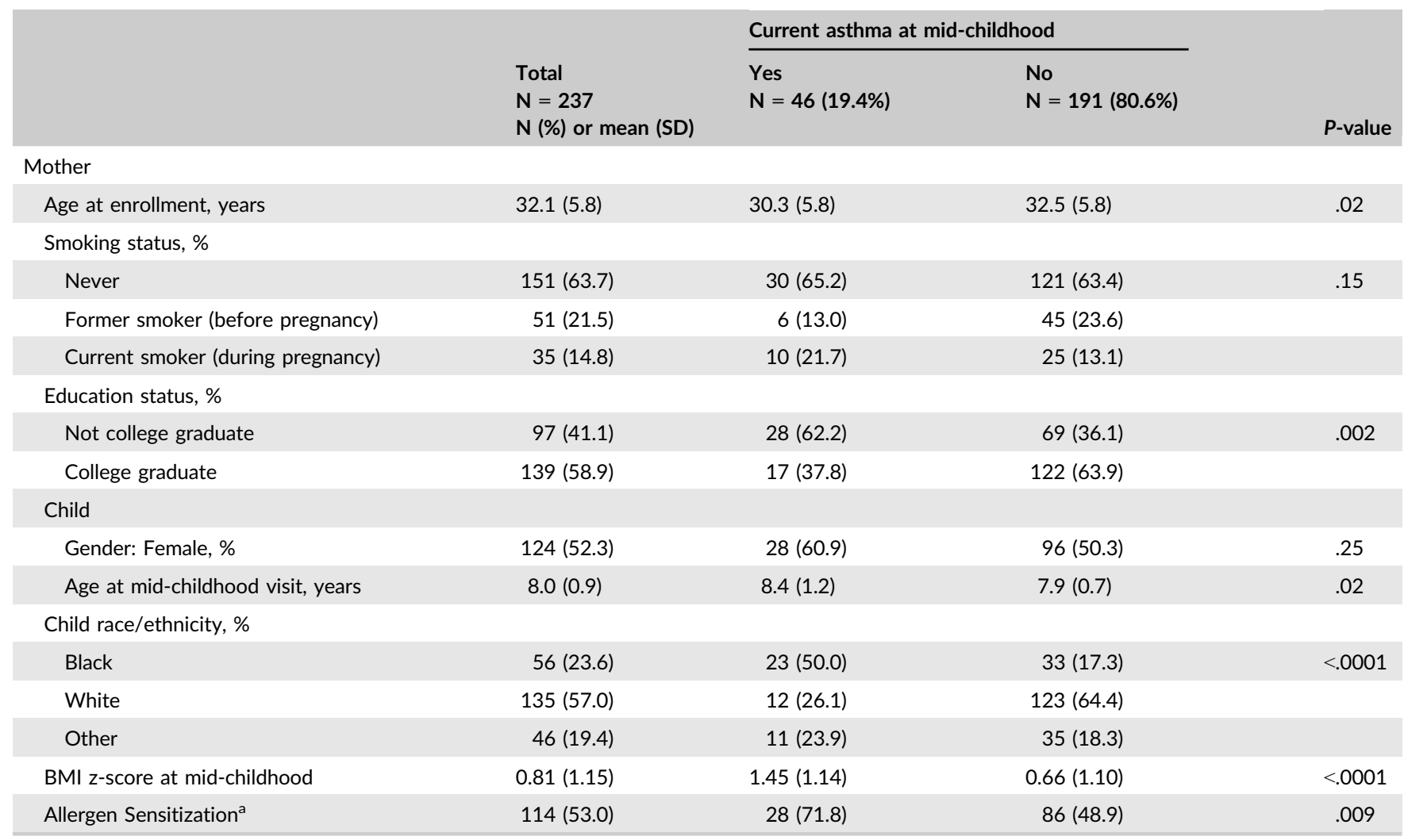

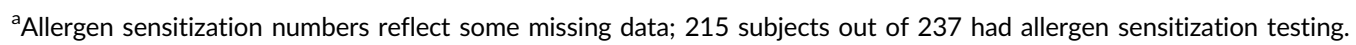

( \pm SD) age was $8.0( \pm 0.9)$ years. The majority of children with current asthma were also sensitized to allergens. Seventy-two percent had a positive specific IgE test $(\geq 0.35 \mathrm{IU} / \mathrm{mL})$ to at least one of the specific indoor, outdoor or food allergens tested. In contrast, $49 \%$ of control subjects were sensitized to allergens. There were also notable differences between children with vs. without current asthma with respect to maternal age (30.3 vs 32.5 years, $P=.02$ ) and education $(37.8 \%$ vs $63.9 \%$ college graduates, $P=.002)$ and child age (8.4 vs 7.9 years, $P=.02)$, race/ethnicity $(26.1 \%$ vs $64.4 \%$ white, $P \leq .0001)$ and BMI $z$-scores (1.45 vs $0.66, P<.0001)$. There were no differences between children with vs without current asthma with respect to child's gender or maternal pregnancy smoking status.

We also collected information on asthma medication use and asthma severity in children with current asthma. The vast majority (80\%) of current asthmatics were taking inhaled corticosteroids. In general, most current asthmatics in Project Viva showed mild to moderate disease severity. None of the current asthmatics were hospitalized in the past year for asthma. Most children with current asthma did not require emergency treatment for asthma symptoms in the past year (72\%), although $17 \%$ reported one emergency room visit for asthma, 9\% reported visiting the emergency room 2-3 times in the past year, with $2 \%$ reporting 3 or more visits. A little over half of the children (58\%) with current asthma occasionally missed school because of asthma symptoms; $20 \%$ report one missed school day, $26 \%$ report 2-3 missed school days, and $11 \%$ report more than three missed school days over a 1 year time period.
In VDAART, cases of current asthma were generally mild. Only $38 \%$ of children received any medication for wheezing, wheezy bronchitis or asthma since their last follow-up. Seventeen percent of current asthmatics report seeing a doctor because of wheezing, asthma, wheezing or asthmatic bronchitis since their last follow-up.

\section{2 | Replication population}

Of the 411 children with available metabolomics data in the VDAART population, 219 (53\%) were male and 108 (26\%) developed asthma by age three. VDAART participants were of diverse race/ethnic backgrounds, and were 48\% African American, 33\% white and $19 \%$ other race. Of the children with current asthma in VDAART, $58 \%$ were sensitized to at least one of the indoor, outdoor or food allergens tested, while approximately half $(48 \%)$ of the control subjects were sensitized to allergens.

\subsection{Partial least squares discriminant analysis}

There was no evidence in these analyses that a specific profile signature derived from the 345 identified metabolites differed between asthma cases and controls in Project Viva (Figure S1). After sevenfold internal cross-validation accuracy was 0.8 , but the $R^{2}$ and $Q^{2}$ were only 0.25 and 0.05 , respectively, for the first component, indicating that the model was not robust. This was confirmed by the permutation testing (permuted $P=.134$ ). 


\section{4 | Individual metabolite analysis}

Of all of the metabolites tested in Project Viva for associations with current asthma case status, none were statistically significant after adjustment for multiple comparisons. However, 10 metabolites were nominally significant at the $P<.05$ level (Table 2 ). A one unit increase in N1-Methyl-2-pyridone-5-carboxamide, a metabolite in the nicotinamide pathway, was associated with an increased odd of asthma $(O R=2.79,95 \% \mathrm{Cl} 1.10-7.97)$. The relationship between N1-Methyl-2-pyridone-5-carboxamide and asthma case status was replicated (Table 3 ) in the VDAART cohort (OR $=1.27, P=.04$ (onesided $P$ value)). The pyrimidine metabolite 5,6-dihydrothymine was associated with reduced odds of asthma $(\mathrm{OR}=0.44,95 \% \mathrm{Cl} 0.21$ 0.86) in Project Viva (Table 2), but showed the opposite direction of effect in VDAART (Table 3). Metabolomics profiling also uncovered two peptides (DSGEGDFXAEGGGVR and ADSGEGDFXAEGGGVR) associated with current asthma in Project Viva. An NCBI protein blast search revealed fibrinopeptide $A$ as the top hit (highest maximum alignment score and lowest $E$ value) for these peptides. Bile constituents taurocholate and biliverdin were also related to asthma case status. Taurocholate, a bile salt involved in fat emulsification, was associated with current asthma (OR $=2.03,95 \% \mathrm{Cl} 1.22-3.42)$, while biliverdin, a bile pigment and product of heme catabolism was associated with decreased odds of current asthma (OR $=0.3595 \%$ $\mathrm{Cl}$ 0.14-0.87). p-cresol sulphate, a bacterial metabolite originating from the gut microbiome, was linked to decreased odds of current asthma (OR $=0.47,95 \% \mathrm{Cl} 0.23-0.94)$. This association was replicated in the VDAART study (Table 3). Other metabolites demonstrating lower levels amongst asthmatic cases in Project Viva were cortisone (a naturally occurring glucocorticoid), tryptophan betaine (from dietary intake of legumes), and 1-docosapentaenoylglycerophosphocholine (22:5), a lysophosphospholipid formed by hydrolysis of phosphatidylcholine by phospholipase A2. Spectra for the two metabolites associated with current asthma in both the discovery and replication cohort (N1-Methyl-2-pyridone-5-carboxamide and p-cresol sulphate) are shown in Figures S2 and S3.
Based on prior reports, we looked to see if plasma cortisone was lower in current asthmatics on ICS as compared to untreated current asthmatics in Project Viva. We did not see an association between ICS use and lower cortisone levels in current asthma cases $(P>.9$ for $t$-test comparisons). However, the very high ICS treatment rates in current asthmatics (80\%) meant that a comparison with untreated current asthmatics was likely underpowered.

\section{DISCUSSION}

We identified metabolites associated with asthma by profiling the plasma metabolome in children from two longitudinal birth cohort studies. In Project Viva, current asthma was associated with alterations in metabolites from the nicotinamide pathway, pyrimidine metabolism, fibrinogen-associated peptides, bile metabolites and p-cresol sulphate, a microbial metabolite derived from the gut microbiome. Cortisone, a metabolite inversely associated with ICS use, ${ }^{31}$ was decreased in current asthmatics. The association between asthma case status and two of the top metabolites (the bacterial metabolite $p$-cresol sulphate from the gut microbiome, and a nicotinoamide pathway metabolite) were replicated in a second cohort, the VDAART clinical trial.

Our study shows a new potential link between higher levels of plasma $\mathrm{p}$-cresol sulphate and decreased odds of asthma. This association was replicated in an independent cohort. p-cresol sulphate is a microbial metabolite that may reflect gut microbiome composition. ${ }^{32}$ Gut microbes have long been hypothesized to interact with the immune system in ways that may alter risk of allergies and asthma, and a number of publications now show compositional differences in the gut microbiome when allergic disease risk factors or allergic disease phenotypes are present. $^{33-38}$ It has been hypothesized that $\mathrm{p}$-cresol sulphate may indicate a gut microbiome enterotype dominated by Bacteroides, ${ }^{32}$ a genera containing multiple taxa including B. Fragilis, a species with strong experimental evidence for restoring Th1/Th2 balance. ${ }^{39}$ While our observed association between $\mathrm{p}$-cresol sulphate

TABLE 2 Metabolites associated with Current Asthma in Project Viva

\begin{tabular}{|c|c|c|}
\hline Pathway/Grouping & Metabolite & $\begin{array}{l}\text { Odds Ratio }(95 \% \mathrm{Cl}) \text { for } \\
\text { Current Asthma }\end{array}$ \\
\hline Nicotinate and Nicotinamide Metabolism & N1-Methyl-2-pyridone-5-carboxamide & $2.79(1.10-7.97)$ \\
\hline Bile Constituents & Biliverdin & $0.35(0.14-0.87)$ \\
\hline Pyrimidine Metabolism & 5,6-dihydrothymine & $0.44(0.21-0.86)$ \\
\hline (Putative) Fibrinopeptide A & ADSGEGDFXAEGGGVR ${ }^{a}$ & $1.68(1.04-2.73)$ \\
\hline Dietary Metabolites & Tryptophan Betaine & $0.47(0.28-0.77)$ \\
\hline Endogenous steroids & Cortisone & $0.20(0.06-0.59)$ \\
\hline Glycerophospholipid metabolism & 1-docosapentaenoylglycerophosphocholine (22:5) & $0.44(0.22-0.88)$ \\
\hline
\end{tabular}

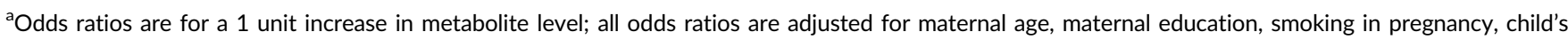
BMI z-score, race/ethnicity, age and gender; Odds ratios with $P<.05$ in bold. 
TAB LE 3 Replication of Significant Metabolites Associated with Current Asthma in VDAART Population

\begin{tabular}{|llll|} 
Pathway/Grouping & Metabolite & Odds Ratio (95\% Cl) for Asthma & $\begin{array}{c}P \text { value (one sided) } \\
\text { for Replication }\end{array}$ \\
\hline Nicotinate and Nicotinamide Metabolism & N1-Methyl-2-pyridone-5-carboxamide & $1.27(0.97-1.66)$ & .04 \\
\hline Bile Constituents & Taurocholate & $1.03(0.89-1.20)$ & .35 \\
\hline Microbial Metabolite & Biliverdin & $1.08(0.90-1.31)$ & .20 \\
\hline Pyrimidine Metabolism & p-cresol sulphate & $0.83(0.69-1.00)$ & .03 \\
\hline Dietary Metabolite & 5,6-dihydrothymine & $1.56(1.00-2.40)$ & .02 \\
\hline Endogenous steroids & Tryptophan Betaine & $1.01(0.92-1.12)$ & .38 \\
\hline
\end{tabular}

aModels adjusted for maternal age, maternal education, child's BMI, race/ethnicity, age and gender.

Odds ratio that replicates in the same direction of effect with $P<.05$ is in bold.

and asthma status is intriguing, further research is required to uncover the connection between the gut microbiome, the circulating metabolome, and their combined relationship to asthma.

We observed higher levels of the nicotinamide pathway metabolite N1-Methyl-2-pyridone-5-carboxamide in asthmatic subjects vs. controls in Project Viva, and our findings in VDAART were showed a similar relationship. The nicotinate and nicotinamide metabolic pathway has been linked to asthma phenotypes in both in vitro and population-based studies. One in vitro study of human airway smooth muscle demonstrated that cells from asthmatic donors had increased expression of NADPH (Nicotinamide Adenine Diphosphate) Oxidase, which promoted oxidative stress and smooth muscle cell contractility. ${ }^{40}$ Other in vitro findings show increased NADPH oxidase expression in neutrophilic asthma, with a concomitant decrease in ciliary function of the bronchial epithelium. ${ }^{41}$ These in vitro models provide a biological basis for our observed association between plasma nicotinamide metabolites and current asthma. While one other previous epidemiological study reported alterations in nicotinamide pathway metabolites (nicotinamide was increased in asthmatic subjects vs. controls), the sample size was small (30 subjects in total) and there were no attempts at replication. ${ }^{42}$

Metabolomic profiles of asthmatics in our study also showed increased levels of two similar peptides likely derived from fibrinopeptide $A$ (DSGEGDFXAEGGGVR and ADSGEGDFXAEGGGVR). Fibrinopeptide $A$ is a short amino acid sequence located within the alpha chain of soluble fibrinogen. Fibrinogen may play a role in asthma pathogenesis by enhancing inflammatory response. Huang and colleagues reported a correlation between increased plasma fibrinogen and reduced lung function. These investigators also found that obese subjects, including those with asthma, tend to have higher circulating fibrinogen levels. ${ }^{43}$ Fibrinogen cleavage products act as TLR4 ligands, enhancing innate immune response. ${ }^{44}$ For instance, fibrinogen cleavage products can bind Toll like receptor 4 (TLR4), priming innate immune and airway epithelial cell response to IL-13, with downstream triggering of airway inflammation. ${ }^{45}$

Two of the metabolites with altered levels in current asthmatics, taurocholate and biliverdin, are bile constituents. Taurocholate, a conjugate of taurine and cholic acid, is an emulsifier of fats. Comhair and investigators previously reported increased plasma taurocholate in asthmatics, and we replicate that finding here in a much larger cohort. ${ }^{42} \mathrm{Yu}$ and colleagues also report altered levels of taurocholate in a mouse model of OVA-induced asthma ${ }^{46}$; however, in this experimental study mice with the asthma phenotype had lower, as opposed to higher, levels of circulating taurocholate. Other experimental data suggest that activity of hemoxygenase-1, the enzyme that produces biliverdin from heme, may reduce mucous secretion in the airways. In an in vitro study of normal human bronchial epithelial cells, over-expression of heme oxygenase-I was associated with reduced IL-13 induced goblet cell hyperplasia and decreased MUCA5 secretion. ${ }^{47}$ The lower levels of circulating biliverdin in asthma cases may reflect reduced activity of heme oxygenase I, which could potentially contribute to increased mucous production in the airways (a common feature of asthma).

Consistent with the findings of Reinke et $\mathrm{al}^{31}$ we also observed decreased plasma cortisone levels in subjects with current asthma. This finding is consistent with well-known effects of ICS (inhaled corticosteroid) use, which cause suppression of the hypothalamic-pituitary axis, leading to lower production of endogenous steroids like cortisone. ${ }^{48}$ The decreased plasma cortisone levels in asthmatics vs. controls is most likely a simple reflection of ICS use, and not an indicator of asthma pathophysiology. Since the majority of asthmatics were treated with ICS, we could not disentangle the effects of ICS use from those associated with current asthma status in our analysis.

Lastly, our observation that pyrimidine metabolism may be altered in asthmatics vs. controls may have relevance for asthma pathophysiology. A large metabolomics study in a cohort of childhood asthmatics demonstrated associations between pyrimidine metabolites and three phenotypic aspects of asthma (methacholine responsiveness, pre bronchodilator FEV1/FVC ratio and post bronchodilator FEV1/FVC ratio). ${ }^{49}$ However the mechanistic connection between pyrimidine metabolism and physiological processes influencing asthma remains unclear. Findings for our discovery population and replication cohort demonstrated opposite directions of effect for the 5,6-dihydorthymine metabolite, an inconsistency that makes the associations difficult to interpret.

It is interesting to note that our PLS-DA analysis did not identify any global metabolite profile differences by asthma status, whereas analysis of individual metabolites did yield a number of biologically plausible associations. One interpretation of this finding is that asthma case 
status is associated with perturbations of few individual metabolites, rather than latent variables describing global pathway alterations (shifts in multiple correlated metabolites). An alternate explanation is that, even with hundreds of named metabolites in our analysis, we still did not have a broad enough coverage of underlying correlated metabolites and their associated pathways to detect global metabolome shifts associated with asthma case status. Instead, we may have identified individual, surrogate metabolite markers of more global pathway perturbations, through our logistic regression analyses. As metabolite identifications continue to expand, PLS-DA may be a more effective tool in identifying global shifts in the metabolome of complex diseases.

Our study has several strengths. First, it is one of the largest plasma metabolomic studies comparing asthma cases to control subjects to date. Second, we limited potential sources of bias by controlling for confounders, including race, BMI and maternal educational level. Third, we were able to replicate two of our top metabolites in an independent cohort. Despite the strengths of our study, a few caveats deserve mention. First, utilizing maternal report of doctor diagnosis of asthma, rather than direct physician report of asthma diagnosis may have been potential source of bias in health outcome assessment. Second, while our study is large compared to other metabolomics studies, our sample size may have been too small to identify associations that were statistically significant after accounting for multiple comparisons. An additional limitation was that the phenotypes available for our discovery and replication cohorts were different. For Project Viva, current asthma status was assessed in mid-childhood, whereas VDAART subjects were assessed at age 3 , when a definitive asthma diagnosis is often difficult. Also, the racial/ethnic and socioeconomic status distributions (while accounted for in our analyses) and different geographic locations for the primary and replication populations, may have given rise to very different exposure profiles (including diet) that could not be adjusted for in our analyses. Plasma samples in Project Viva were fasting, whereas in the replication population plasma samples were non-fasting. (The use of non-fasting samples in VDAART may have had a particular influence on our ability to detect an association between current asthma status and the dietary metabolite tryptophan betaine, if recent food intake increased the variability of this metabolite.) The study designs for Project Viva and VDAART were also different; Project Viva is an observational epidemiology study based on an unselected population, while VDAART is a clinical trial of prenatal vitamin D supplementation and asthma outcomes in children with a parental history of allergies or asthma. These discrepancies in the two cohorts may have decreased our potential for replication; however, our ability to replicate two metabolites in spite of these differences suggests that our replicated associations are robust. Metabolite identification in the two populations was not identical, which meant that we did not have the opportunity to test for replication of the phospholipid 1-docosapentaenoylglycerophosphocholine (22:5) and fibrinogen peptide findings, as these were not among the named metabolites in our replication study. It should also be noted that while many of the metabolites associated with asthma in our primary cohort are biologically plausible and supported by experimental data in the literature, none were statistically significant after adjustment for multiple comparisons.
In summary, our findings suggest that current asthma is associated with perturbations in pathways including metabolism of nicotinamide and pyrimidines, production of bile salts, heme catabolism and metabolites generated via the gut microbiome. Asthmatics in our cohort tended to have lower levels of endogenous cortisone, which is likely an effect of inhaled corticosteroid use. Identifying a metabolomic profile characteristic of children with asthma has the potential to uncover novel candidate pathways in asthma pathophysiology. In the long-term, asthma metabolomics research may identify biomarkers that improve the efficacy of therapeutic regimens for children with asthma, in turn decreasing the severity of asthma suffering and healthcare costs in the United States and globally.

\section{ACKNOWLEDGEMENTS}

This work was supported by the National Institutes of Health [K24 HD069408, R01Al102960, R01 HD034568, U01HL091528].

\section{CONFLICT OF INTEREST}

The authors have no conflicts of interest.

\section{ORCID}

\section{R. S. Kelly (iD http://orcid.org/0000-0003-3023-1822 \\ J. E. Sordillo (D) http://orcid.org/0000-0002-4966-1796}

\section{REFERENCES}

1. Barnett SB, Nurmagambetov TA. Costs of asthma in the United States: 2002-2007. J Allergy Clin Immunol. 2011;127:145-152.

2. Rava M, Smit LA, Nadif R. Gene-environment interactions in the study of asthma in the postgenomewide association studies era. Curr Opin Allergy Clin Immunol. 2015;15:70-78.

3. Bonnelykke $\mathrm{K}$, Ober $\mathrm{C}$. Leveraging gene-environment interactions and endotypes for asthma gene discovery. J Allergy Clin Immunol. 2016;137:667-679.

4. Moffatt MF, Gut IG, Demenais F, et al. A large-scale, consortiumbased genomewide association study of asthma. N Engl J Med. 2010;363:1211-1221.

5. Rhee EP, Gerszten RE. Metabolomics and cardiovascular biomarker discovery. Clin Chem. 2012;58:139-147.

6. Wang TJ, Larson MG, Vasan RS, et al. Metabolite profiles and the risk of developing diabetes. Nat Med. 2011;17:448-453.

7. Rhee EP, Thadhani R. New insights into uremia-induced alterations in metabolic pathways. Curr Opin Nephrol Hypertens. 2011;20:593-598.

8. Mapstone M, Cheema AK, Fiandaca MS, et al. Plasma phospholipids identify antecedent memory impairment in older adults. Nat Med. 2014;20:415-418.

9. Carraro S, Rezzi S, Reniero F, et al. Metabolomics applied to exhaled breath condensate in childhood asthma. Am J Respir Crit Care Med. 2007;175:986-990.

10. Saude EJ, Skappak CD, Regush S, et al. Metabolomic profiling of asthma: diagnostic utility of urine nuclear magnetic resonance spectroscopy. J Allergy Clin Immunol. 2011;127:6.

11. Sinha A, Krishnan V, Sethi $T$, et al. Metabolomic signatures in nuclear magnetic resonance spectra of exhaled breath condensate identify asthma. Eur Respir J. 2012;39:500-502. 
12. Mattarucchi E, Baraldi E, Guillou C. Metabolomics applied to urine samples in childhood asthma; differentiation between asthma phenotypes and identification of relevant metabolites. Biomed Chromatogr. 2012;26:89-94.

13. Carraro S, Giordano G, Reniero F, et al. Asthma severity in childhood and metabolomic profiling of breath condensate. Allergy. 2013;68:110117.

14. Gahleitner F, Guallar-Hoyas C, Beardsmore CS, Pandya HC, Thomas CP. Metabolomics pilot study to identify volatile organic compound markers of childhood asthma in exhaled breath. Bioanalysis. 2013;5:2239-2247.

15. Jung J, Kim SH, Lee HS, et al. Serum metabolomics reveals pathways and biomarkers associated with asthma pathogenesis. Clin Exp Allergy. 2013;43:425-433

16. Ibrahim B, Marsden P, Smith JA, Custovic A, Nilsson M, Fowler SJ. Breath metabolomic profiling by nuclear magnetic resonance spectroscopy in asthma. Allergy. 2013;68:1050-1056.

17. Ried JS, Baurecht $\mathrm{H}$, Stuckler $\mathrm{F}$, et al. Integrative genetic and metabolite profiling analysis suggests altered phosphatidylcholine metabolism in asthma. Allergy. 2013;68:629-636.

18. Loureiro CC, Duarte IF, Gomes J, et al. Urinary metabolomic changes as a predictive biomarker of asthma exacerbation. J Allergy Clin Immunol. 2014;133:5.

19. McGeachie MJ, Dahlin A, Qiu W, et al. The metabolomics of asthma control: a promising link between genetics and disease. Immun Inflamm Dis. 2015;3:224-238.

20. Chang C, Guo ZG, He B, Yao WZ. Metabolic alterations in the sera of Chinese patients with mild persistent asthma: a GC-MS-based metabolomics analysis. Acta Pharmacol Sin. 2015;36:1356-1366.

21. Smolinska A, Klaassen EM, Dallinga JW, et al. Profiling of volatile organic compounds in exhaled breath as a strategy to find early predictive signatures of asthma in children. PLoS ONE. 2014;9:e95668.

22. Fitzpatrick AM, Park Y, Brown LA, Jones DP. Children with severe asthma have unique oxidative stress-associated metabolomic profiles. Journal of Allergy and Clinical Immunology. 2013;133:261.e8.

23. Oken E, Baccarelli AA, Gold DR, et al. Cohort profile: project viva. Int J Epidemiol. 2015;44:37-48.

24. Perng W, Gillman MW, Fleisch AF, et al. Metabolomic profiles and childhood obesity. Obesity (Silver Spring). 2014;22:2570-2578.

25. Kuczmarski RJ, Ogden CL, Guo SS, et al. 2000 CDC Growth Charts for the United States: methods and development. Vital Health Stat 11. 2002;246:1-190.

26. Ryals J, Lawton K, Stevens D, Milburn M. Metabolon, Inc. Pharmacogenomics. 2007;8:863-866.

27. Litonjua AA, Lange NE, Carey VJ, et al. The Vitamin D Antenatal Asthma Reduction Trial (VDAART): rationale, design, and methods of a randomized, controlled trial of vitamin $\mathrm{D}$ supplementation in pregnancy for the primary prevention of asthma and allergies in children. Contemporary clinical trials. 2014;38:37-50.

28. Litonjua AA, Carey VJ, Laranjo N, Harshfield BJ, et al. Effect of Prenatal Supplementation With Vitamin D on Asthma or Recurrent Wheezing in Offspring by Age 3 Years The VDAART Randomized Clinical Trial. JAMA. 2016;315:362-370.

29. $R$ Development Core Team. $R$ : A language and environment for statistical computing. Vienna, Austria: R Development Core Team; 2017.

30. Xia J, Sinelnikov IV, Han B, Wishart DS. MetaboAnalyst 3.0-making metabolomics more meaningful. Nucleic Acids Res. 2015;43:251.

31. Reinke SN, Gallart-Ayala H, Gomez C, et al. Metabolomics analysis identifies different metabotypes of asthma severity. Eur Respir J. 2017;49:2016.

32. Viaene L, Thijs L, Jin Y, et al. Heritability and clinical determinants of serum indoxyl sulfate and $p$-cresyl sulfate, candidate biomarkers of the human microbiome enterotype. PLoS ONE. 2014;9:e79682.

33. Edwards CA. Determinants and duration of impact of early gut bacterial colonization. Ann Nutr Metab. 2017;70:246-250.
34. Johnson CC, Ownby DR. The infant gut bacterial microbiota and risk of pediatric asthma and allergic diseases. Transl Res. 2017;179:60-70.

35. Sordillo JE, Zhou Y, McGeachie MJ, et al. Factors influencing the infant gut microbiome at age 3-6 months: Findings from the ethnically diverse vitamin $\mathrm{D}$ antenatal asthma reduction trial (VDAART). J Allergy Clin Immunol. 2017;139:491.e14.

36. Fujimura KE, Sitarik AR, Havstad S, et al. Neonatal gut microbiota associates with childhood multisensitized atopy and T cell differentiation. Nat Med. 2016;22:1187-1191.

37. Arrieta MC, Stiemsma LT, Dimitriu PA, et al. Early infancy microbial and metabolic alterations affect risk of childhood asthma. Sci Transl Med. 2015;7:307ra152.

38. Johnson CC, Ownby DR. The infant gut bacterial microbiota and risk of pediatric asthma and allergic diseases. Transl Res. 2017;179:60-70.

39. Mazmanian SK, Liu CH, Tzianabos AO, Kasper DL. An immunomodulatory molecule of symbiotic bacteria directs maturation of the host immune system. Cell. 2005;122:107-118.

40. Sutcliffe A, Hollins F, Gomez E, et al. Increased nicotinamide adenine dinucleotide phosphate oxidase 4 expression mediates intrinsic airway smooth muscle hypercontractility in asthma. Am J Respir Crit Care Med. 2012;185:267-274.

41. Wan WY, Hollins F, Haste L, et al. NADPH oxidase-4 overexpression is associated with epithelial ciliary dysfunction in neutrophilic asthma. Chest. 2016;149:1445-1459.

42. Comhair SA, McDunn J, Bennett C, Fettig J, Erzurum SC, Kalhan SC Metabolomic endotype of asthma. J Immunol. 2015;195:643-650.

43. Huang F, del-Rio-Navarro BE, Alcantara ST, et al. Plasminogen activator inhibitor-1, fibrinogen, and lung function in adolescents with asthma and obesity. Endocr Res 2012;37:135-144.

44. Millien VO, Lu W, Mak G, et al. Airway fibrinogenolysis and the initiation of allergic inflammation. Ann Am Thorac Soc. 2014;11(Suppl 5):277.

45. Millien VO, Lu W, Shaw J, et al. Cleavage of fibrinogen by proteinases elicits allergic responses through Toll-like receptor 4 Science. 2013;341:792-796.

46. Yu M, Cui FX, Jia HM, et al. Aberrant purine metabolism in allergic asthma revealed by plasma metabolomics. J Pharm Biomed Anal. 2016;120:181-189.

47. Mishina K, Shinkai M, Shimokawaji T, et al. HO-1 inhibits IL-13induced goblet cell hyperplasia associated with CLCA1 suppression in normal human bronchial epithelial cells. Int Immunopharmacol. 2015;29:448-453.

48. Kannisto S, Laatikainen A, Taivainen A, Savolainen K, Tukiainen $\mathrm{H}$, Voutilainen R. Serum dehydroepiandrosterone sulfate concentration as an indicator of adrenocortical suppression during inhaled steroid therapy in adult asthmatic patients. Eur J Endocrinol. 2004;150:687-690.

49. Kelly RS, Virkud Y, Giorgio R, Celedon JC, Weiss ST, Lasky-Su J. Metabolomic profiling of lung function in Costa-Rican children with asthma. Biochim Biophys Acta. 2017;1863:1590-1595.

\section{SUPPORTING INFORMATION}

Additional supporting information may be found online in the Supporting Information section at the end of the article.

How to cite this article: Kelly RS, Sordillo JE, Lasky-Su J, et al. Plasma metabolite profiles in children with current asthma. Clin Exp Allergy. 2018;48:1297-1304.

https://doi.org/10.1111/cea.13183 